\title{
Electric Dipole and Current Induced Spin Resonances of Shallow Donor in $\mathrm{ZnO}$
}

\author{
E. Michaluk ${ }^{a}$, J. BŁoniarz ${ }^{a}$, M. Pabich $^{b}$, Z. Wilamowski $^{a, b}$ \\ AND A. MYCIELSKI ${ }^{a}$ \\ ${ }^{a}$ Institute of Physics, Polish Academy of Sciences \\ al. Lotników 32/46, 02-668 Warsaw, Poland \\ ${ }^{b}$ Department of Mathematics and Computer Science, UWM \\ Żołnierska 14, 10-561 Olsztyn, Poland
}

\begin{abstract}
In this paper we present our observation of electric dipole spin resonance and current induced spin resonance in $\mathrm{ZnO}$ bulk crystals, both effects result from spin-orbit coupling. Electric dipole spin resonance originates from admixture of spin state due to spin-orbit interaction which leads to probability of spin-flip caused by electric component of microwave field. In current induced spin resonance phenomenon the electric component of microwave field induces electron motion leading to mean ac spin-orbit field that acts on electron spin. Spin-orbit interaction can be described in terms of the Rashba field, an effective magnetic field that induce spin resonance. In contrast to magnetic and electric dipole spin resonances the current induced resonance is characterized by dispersive-like line shape.
\end{abstract}

PACS numbers: 71.55.Gs, 76.30.--v, 71.70.Ej

\section{Introduction}

Spin-orbit (SO) coupling plays a crucial role in understanding and design of modern spintronics devices. It allows manipulating carrier spin by means of electric field only. It is well known that strength of spin-orbit interaction in two-dimensional electron gas channel can be controlled by a gate voltage. These, as well as many others, spin related features open a new possibility in creation of functional spin devices such as spin field effect transistor or spin filter. In systems without inversion symmetry, due to spin-orbit interaction, it is possible to induce shift of spin resonance of conducting electrons by applying high current density. It is also possible to induce spin flip transitions between electron states that are normally forbidden. $\mathrm{ZnO}$, due to wurtzite structure, is an example of such a system where spin-orbit coupling leads to many interesting phenomena. 


\section{Samples and experimental setup}

The $\mathrm{ZnO}$ bulk crystals were grown in closed quartz ampoule using the chemical vapor transport (CVT). Pure $\mathrm{ZnO}$ was synthesized at $650^{\circ} \mathrm{C}$, by reaction between high purity $\mathrm{ZnO}(6 \mathrm{~N})$ and spectrally pure oxygen. The crystal growth took place in a graphite covered quartz ampoule. The mixtures: $\mathrm{H}_{2}+\mathrm{C}+\mathrm{H}_{2} \mathrm{O}$ or $\mathrm{N}_{2}+\mathrm{C}+\mathrm{H}_{2} \mathrm{O}$ were used as transporting chemical agents. Polycrystals obtained by this method have the averaged volume of about $3 \mathrm{~cm}^{3}$ and consist of monocrystal grains of $0.2-0.5 \mathrm{~cm}^{3}$. As grown crystals were red colored (irrespective of chemical agents used), and after annealing in oxygen atmosphere at $900-1000^{\circ} \mathrm{C}$ the crystals became colorless. In this work we investigated both as grown and annealed crystals. From ESR measurements we estimate the donor concentration to be about $10^{17} \mathrm{~cm}^{-3}$ for as grown crystals and this is much smaller than concentration of color centers estimated on the basis of intensity of red color of the sample. After annealing in oxygen atmosphere at $900-1000^{\circ} \mathrm{C}$, the crystals become colorless while the electron concentration (determined from ESR amplitude) decreases only one order of magnitude. Therefore we conclude that ESR signal cannot be related to color centers. The origin of the native donors has not been identified. Since the crystals were not intentionally doped, the donors may result from native defects associated with either oxygen vacancies or zinc interstitials.

The electric dipole spin resonance (EDSR) and current induced spin resonance (CISR) measurements were performed on Bruker ESP-300 spectrometer (standard commercial setup) at $9.5 \mathrm{GHz}$ (X-band). The experiments were performed in longitudinal Voigt geometry. In this configuration the propagation of the microwave is perpendicular to the homogeneous external magnetic field $\boldsymbol{H}_{0}$ and microwave electric field $\boldsymbol{E}_{1}$ is always parallel to $\boldsymbol{H}_{0}$. The $\mathrm{TE}_{102}$ mode cavity was used for which standing electromagnetic wave has the maximum magnetic field component, $\boldsymbol{H}_{1}$, in the center of the cavity (whereas $\boldsymbol{E}_{1}$ is zero). Therefore in the middle of the cavity the sample should interact only with magnetic field component which oscillates with frequency of about 9.5 GHz. The electric field component that oscillates with the same frequency may appear either due to displacement of the sample from central position or perturbation of standing wave by the sample. In ESR measurements, $c$ axis of the crystal was oriented at the angle $\alpha$ with respect to $\boldsymbol{E}_{1}$ and $\boldsymbol{H}_{0}$. The $\alpha$ varied in range $0^{\circ}-360^{\circ}$.

\section{The Rashba field and $g$-factor anisotropy}

In principle in wurtzite structure the spin-orbit Hamiltonian is proportional to the vector product of electron velocity $\boldsymbol{v}$ and intrinsic, symmetry breaking, crystal electric field $\boldsymbol{E}_{\mathrm{c}}: \hat{H}_{\mathrm{SO}} \propto\left(\boldsymbol{v} \times \boldsymbol{E}_{\mathrm{c}}\right) \cdot \boldsymbol{s}$, where $\boldsymbol{s}$ denotes spin of the electron. Such interaction can be described in terms of effective Zeeman Hamiltonian $\hat{H}_{\mathrm{R}}=$ $g \mu_{\mathrm{B}} \boldsymbol{s} \cdot \boldsymbol{H}_{\mathrm{R}}$, where

$$
\boldsymbol{H}_{\mathrm{R}}=\frac{\alpha_{\mathrm{R}}}{g \mu_{\mathrm{B}}}\left(\boldsymbol{v} \times \boldsymbol{E}_{\mathrm{c}}\right)
$$


is so-called magnetic Rashba field and $\alpha_{\mathrm{R}}$ is the Rashba coefficient which depends on a strength of spin-orbit coupling. This field acts on spin of each electron irrespective of the external magnetic field $\boldsymbol{H}_{0}$. The effective magnetic field which affects electron spin is a vector sum $\boldsymbol{H}=\boldsymbol{H}_{0}+\boldsymbol{H}_{\mathrm{R}}$. The Rashba field is perpendicular to both, the momentum of the electron, $\hbar \boldsymbol{k}$ and the direction of symmetry breaking built-in electric field $\boldsymbol{E}_{\mathrm{c}}$. In finite temperature charge carriers have always thermal velocity directly related to their thermal energy. In three-dimensional system vectors of electron velocity are uniformly distributed in each direction and form a sphere in $\boldsymbol{k}$-space centered in $\boldsymbol{k}=\mathbf{0}$. Only this component of velocity vector which is perpendicular to $c$-axis $\left(\boldsymbol{v}_{\perp}\right)$ contributes to the Rashba field creation. Obviously there is only one plane perpendicular to $c$-axis so it is convenient to identify it as the Rashba plane. All $\boldsymbol{H}_{\mathrm{R}}$ vectors lie on this plane as well as all $\boldsymbol{v}_{\perp}$ vectors (but still $\boldsymbol{H}_{\mathrm{R}} \perp \boldsymbol{v}_{\perp}$, see Fig. 1). The orientation of the Rashba plane does not depend on orientation of external magnetic field so, in particular, it is possible to apply $\boldsymbol{H}_{0}$ perpendicular or parallel to the Rashba plane. These two non-equivalent orientations are shown in Fig. 1.

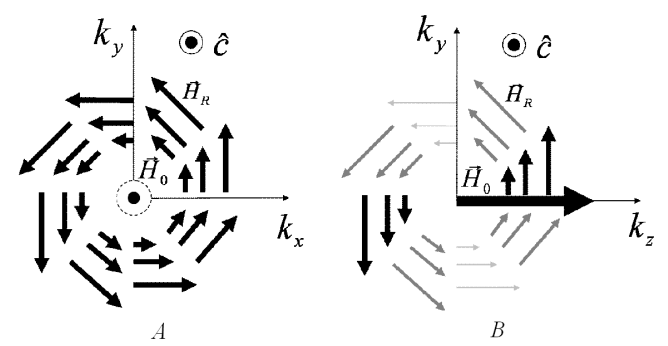

Fig. 1. The Rashba field lies in a plane perpendicular to a symmetry lowering $c$-axis. Arrows on $x-y$ plane symbolize the Rashba vectors. (A) In case when $\hat{c} \| \boldsymbol{H}_{0}$ all $\boldsymbol{H}_{\mathrm{R}}$ vectors are perpendicular to $\boldsymbol{H}_{0}$ and give positive contribution to effective magnetic field. (B) In case when $\hat{c} \perp \boldsymbol{H}_{0}$ the contribution to effective magnetic field is weighted depending on angle between $\boldsymbol{H}_{0}$ and $\boldsymbol{H}_{\mathrm{R}}$. The thickness of the arrow indicates the strength of this contribution (the thicker the higher).

In thermal equilibrium mean value of $\boldsymbol{H}_{\mathrm{R}}$ field acting on spin ensemble vanishes since SO fields for electrons with opposite velocity are oriented in opposite directions and their contributions to the total $\boldsymbol{H}_{\mathrm{R}}$ cancel for pairs.

In second order approximation we assume that each electron feels the effective field $|\boldsymbol{H}|=\left|\boldsymbol{H}_{0}+\boldsymbol{H}_{\mathrm{R}}\right|$ independently.

The value of this field is given by simple formula

$$
H=\sqrt{H_{0}^{2}+H_{R}^{2}-2 H_{0} H_{R} \cos \theta}
$$

where $\theta$ is an angle between $\boldsymbol{H}_{0}$ and $\boldsymbol{H}_{\mathrm{R}}$. Effective $H$ field is distributed around its mean value $\langle H\rangle$. Assuming that $H_{\mathrm{R}} \ll H$ and averaging over all possible orientations of $\boldsymbol{H}_{\mathrm{R}}$ in the Rashba plane we obtain the average effective magnetic field [1]: 


$$
\langle H\rangle=H_{0}+\frac{\left\langle H_{\mathrm{R}}^{2}\right\rangle}{4 H_{0}}\left(1+\cos ^{2} \alpha\right),
$$

where $\alpha$ denotes an angle between $\boldsymbol{H}_{0}$ and normal to the Rashba plane. A separation between averaged effective field $\langle H\rangle$ and $H_{0}$ is as follows:

$$
\langle H\rangle-H_{0}=\frac{1}{4} \frac{\left\langle H_{\mathrm{R}}^{2}\right\rangle}{H_{0}}\left(1+\cos ^{2} \alpha\right)
$$

At resonance condition $\langle H\rangle=h \nu / g \mu_{\mathrm{B}} \equiv H^{\text {res }}$ and this is constant since all right hand side parameters are constant. Equation (2) clearly indicates that for $\hat{c} \| \boldsymbol{H}_{0}$ the separation $H^{\text {res }}-H_{0}^{\text {res }}=\frac{1}{2} \frac{\left\langle H_{\mathrm{R}}^{2}\right\rangle}{H_{0}^{\text {res }}}$ is twice the separation for $\hat{c} \perp \boldsymbol{H}_{0}$ : $H^{\text {res }}-H_{0}^{\text {res }}=\frac{1}{4} \frac{\left\langle H_{\mathrm{R}}^{2}\right\rangle}{H_{0}^{\text {res }}}$. Configuration $\hat{c} \| H_{0}$ is schematically depicted in Fig. 1A. In this case all $\boldsymbol{H}_{\mathrm{R}}^{0}$ vectors are perpendicular to $H_{0}$ thus all of them give positive contribution to average effective magnetic field $\langle H\rangle$. It is not the case when $\hat{c} \perp \boldsymbol{H}_{0}$. As it is shown in Fig. 1B in this configuration there is a fraction of $\boldsymbol{H}_{\mathrm{R}}$ vectors which reduces effective magnetic field whereas the others increase it. In extreme case we can consider two Rashba vectors with opposite direction and parallel to $\boldsymbol{H}_{0}$. In such case there is no modification of field at which resonance occurs, i.e. effective vector length modification cancel for this pair. This leads to broadening of $H$ distribution (i.e. to broadening of resonance line) rather than to deviation of $\langle H\rangle$ from $H_{0}$.

In order to investigate the influence of SO interaction on magnetic resonances in $\mathrm{ZnO}$ we performed a systematic study of anisotropy of line width, $g$-factor and ESR intensity. The ESR signals were observed in as grown $\mathrm{ZnO}$ samples. Typical spectrum consists of single narrow line characterized by line width in the range 0.6-2 Gs. The angular dependence of ESR observed at $3.8 \mathrm{~K}$ are shown in Fig. 2.

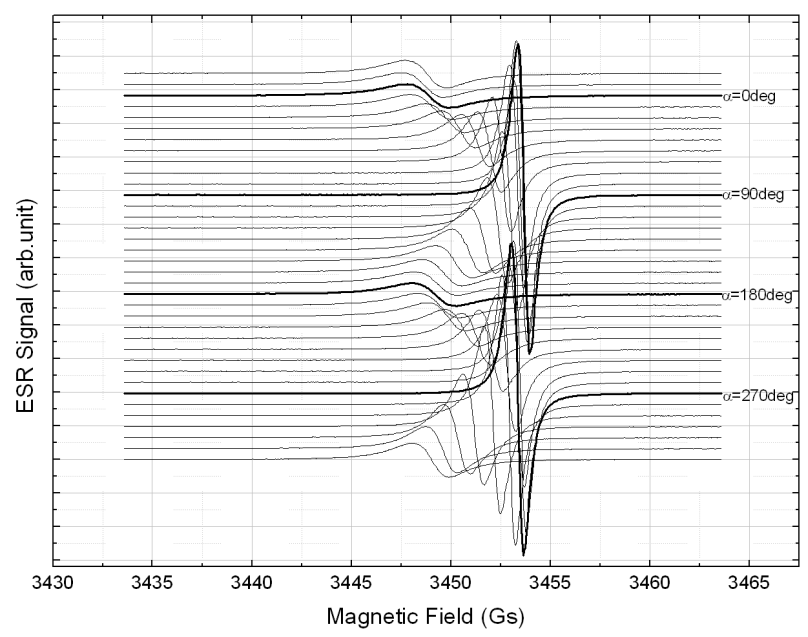

Fig. 2. The angular dependence of electron spin resonance in $\mathrm{ZnO}$ (as grown) wurtzite crystal at $3.8 \mathrm{~K}$ recorded in longitudinal Voigt geometry. Anisotropy of line width, $g$-factor, and ESR intensity is clearly seen. 


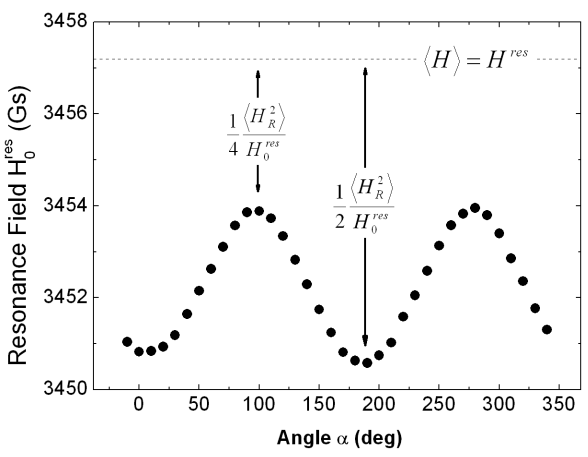

Fig. 3. Resonance line position as a function of angle between $c$-axis and magnetic field $\boldsymbol{H}_{0}$. According to the data shown we evaluated $\sqrt{\left\langle H_{\mathrm{R}}^{2}\right\rangle} \approx 200 \mathrm{Gs}$.

From the data shown in Fig. 2 the dependence of resonance field $\boldsymbol{H}_{0}^{\text {res }}$ (measured external magnetic field at which resonance occurs) on angle $\alpha$ (see Fig. 3) has been found. Assuming that the whole anisotropy of $g$-factor arises from the spin-orbit interaction we estimate $\sqrt{\left\langle H_{\mathrm{R}}^{2}\right\rangle} \approx 200$ Gs from Eq. (2). Since other effects may have their contribution to the $g$-factor anisotropy, the obtained value of the Rashba field is just an upper limit approximation.

\section{Electric dipole spin resonance}

Origin of EDSR was theoretically discussed by Rashba [2] and Rashba and Sheka [3]. They predicted that in many physical systems in which SO interaction mixes states with different symmetry electric-dipole as well as magnetic-dipole transitions can be observed in classical ESR experiment. The former transitions are induced by electric component of electromagnetic radiation. In majority in ESR measurement one uses microwave cavity which is constructed in a way that electric component of standing wave diminishes in a center of the cavity (mode $\mathrm{TE}_{102}$ ). In practice, however, it is not possible to put a sample exactly in a central position so there is always a non-zero electric field component of microwave radiation that acts on a sample. This non-diminishing electric field makes EDSR possible. The presence of electric component of microwave field causes the formation of electric dipoles. Direct transition between two spin states in $1 s$ level is normally forbidden by electric-dipole selection rules. However, these rules are relaxed in crystals in which spin-orbit interaction arises. In this case spin-orbit perturbation mixes states with different orbital angular momentum. In consequence perturbed donor states contain mixture of up and down spins of $2 p$ and $1 s$ level. This effect allows electric-dipole spin-flip transitions within the ground state doublet. After Dobrowolska et al. [4-6] the fingerprint of EDSR is the dependence of ESR intensity on angle $\alpha$. According to selection rules governing electric-dipole transitions $[3-7,1]$ one expects that for longitudinal Voigt geometry and for $\hat{c} \| \boldsymbol{H}_{0}$, i.e. for $\alpha=0^{\circ}$ or $\alpha=180^{\circ}$, electric-dipole transitions cannot 
take place. This geometrical configuration corresponds to minimum amplitude in Fig. 2. Consistently maximum EDSR transition probability is when $\hat{c} \perp \boldsymbol{H}_{0}$, i.e. for $\alpha=90^{\circ}, \alpha=270^{\circ}$, which case corresponds to maximum amplitude in Fig. 2.

Magnetic-dipole transition (usually observed in ESR measurement) produces about $93-60 \%$ of total ESR intensity recorded. The residual signal corresponds to electric-dipole transition. The EDSR signal observed in our experiment is much weaker than that obtained by Dobrowolska. There are two principal reasons for that: (1) construction of our microwave cavity limits the efficiency of electric-dipole transitions (electric component of microwave field diminishes in a sample position), (2) measurements performed by Dobrowolska et al. were taken in much stronger external magnetic field (this aspect is crucial since EDSR transition probability is proportional to $\boldsymbol{H}_{0}^{2}$ ).

\section{Current induced spin resonance}

The EDSR transition between two donor states is not a simple consequence of SO field. Indeed, if one considers the SO interaction in terms of the Rashba field $\hat{H}_{\mathrm{SO}} \propto \boldsymbol{H}_{\mathrm{R}} \cdot \boldsymbol{s}$, where $\hat{\boldsymbol{H}}_{\mathrm{R}} \propto \boldsymbol{v} \times \boldsymbol{E}_{\mathrm{c}}$, it becomes clear that for localized states $\boldsymbol{H}_{\mathrm{R}}$ is equal to zero (since $v$ is zero). Thus studying the influence of SO interaction on electric-dipole transition probabilities for localized states one has to consider second-order contribution [8]. Nevertheless, it is possible to think about SO field as a first order effect. For instance, in case when band electrons are presented, as long as there is external electric field, the average over $k$-states gives non-zero $v$, i.e. there is a current flowing through the sample. In wurtzite structure this yields to a non-vanishing field $\left(\boldsymbol{H}_{\mathrm{R}} \propto \boldsymbol{v} \times \boldsymbol{E}_{\mathrm{c}}\right)$ acting as additional magnetic Rashba field. This extra field may be induced by constant, as well as alternating electric field. In latter case the vectors of electron velocity oscillate following electric field. If the frequency of oscillating electric field is equal to the Larmor frequency, then resultant Rashba field affects electron spin and may give non-negligible contribution to spin resonances. This phenomenon is called current induced spin resonance and was first reported by Wilamowski et al. [9] for two-dimensional electron gas (2DEG) in Si/SiGe heterostructure.

We believe that we observed CISR signal in $\mathrm{ZnO}$ samples annealed in oxygen. For these samples ESR spectrum consists of two narrow lines. Typical ESR spectra recorded at $3.8 \mathrm{~K}$ for different angles $\alpha$ are shown in Fig. 4 . The $\alpha=0$ indicates that $c$-axis is parallel to $\boldsymbol{H}_{0}$. First important feature of the spectra shown in Fig. 4 is a variation of resonance intensity of line $B$ (appearing in higher magnetic field). The ESR amplitude, in case when $\hat{c} \perp \boldsymbol{H}_{0}$, has its maximum, and completely disappears in case when $\hat{c} \| \boldsymbol{H}_{0}$. We suggest that this behavior is a direct consequence of Eq. (1) for microwave cavity mode TE $\mathrm{T}_{102}$. For clarity our cavity realizes longitudinal Voigt geometry thus in all experiments electric component of microwave field is always parallel to $\boldsymbol{H}_{0}$ so that $\boldsymbol{v} \| \boldsymbol{H}_{0}$. When $c$-axis of the crystal is parallel to the external magnetic field $\boldsymbol{H}_{0}$ vector product (1) yields 
zero. In this case resultant SO field diminishes, and thus CISR signal vanishes. This corresponds to the spectrum measured for $\alpha=0^{\circ}$ (see Fig. 4). The same rule indicates that for $\hat{c} \perp \boldsymbol{H}_{0}$ SO field has its maximum and gives rise to the highest amplitude of CISR signal. This configuration corresponds to spectra measured for $\alpha=90^{\circ}$ and $\alpha=-90^{\circ}$.

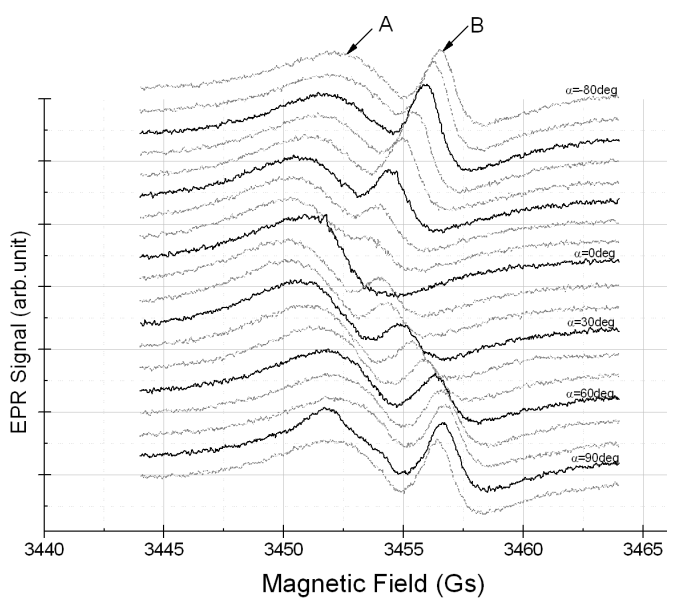

Fig. 4. Angular dependence of electron spin resonance spectra in $\mathrm{ZnO}$ wurtzite crystal recorded at $3.8 \mathrm{~K}$ (all spectra were obtained for longitudinal Voigt geometry).

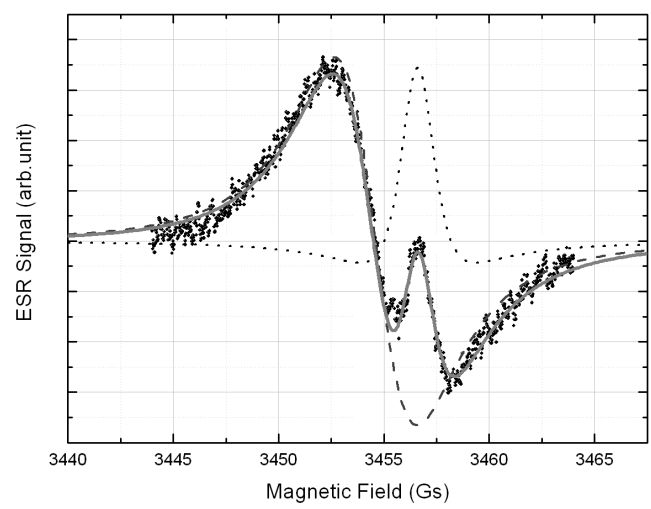

Fig. 5. Example of line decomposition: $a$ - dashed line corresponds to absorption-like and $b$ - dotted line corresponds to dispersive-like component of resonance line (CISR). The thick line is a sum of $a$ and $b$.

Apart from the well pronounced $\alpha$ dependence of signal intensity the most striking feature of the spectra shown in Fig. 4 is an unusual shape of line $B$. Typical ESR line shape for isolated spins is described by the first derivative of Lorentzian function. Line $B$ has dispersion-like character (see Fig. 5). As it 
is shown in Fig. 5 there are two types of signal in general. First line, which appears at $H_{0}=3454.6 \mathrm{Gs}$, is a classical Lorentzian function (dashed line). Second signal which lies slightly below $H_{0}=3457 \mathrm{Gs}$ is dispersive-like (dotted line). We conclude that two mechanisms contribute to measured ESR spectra: classical magnetic dipole transition and current induced spin resonance, depicted as dashed and dotted line, respectively.

\section{Conclusions}

The ESR measurements of undoped $\mathrm{ZnO}$ bulk crystals identify the existence of native shallow donors in concentration $10^{16}$ and $10^{17}$, for annealed and as grown samples, respectively. From angular dependence of ESR line position we estimated the average value of the Rashba field $\sqrt{\left\langle H_{\mathrm{R}}^{2}\right\rangle} \approx 200$ Gs the important parameter describing the strength of spin-orbit coupling. In investigated samples we observed electric-dipole spin resonance and current induced spin resonance both resulting from spin-orbit interaction. EDSR signal appears on a dominant background of magnetic dipole transitions (MDSR). The amplitude of EDSR obeys the selection rules for electric-dipole transition and for $\hat{c} \perp \boldsymbol{H}_{0}$ is 1.54 of amplitude recorded for $\hat{c} \| \boldsymbol{H}_{0}$.

Current induced spin resonance, as distinct from magnetic or electric dipole spin resonance, is characterized by dispersive-like line shape. The dispersive-like character together with measured angular dependence of line intensity are strong arguments for that we indeed found the CISR signal in ZnO bulk crystals.

\section{Acknowledgments}

This work was supported by grant 3T08A 05128.

\section{References}

[1] T. Dietl, J. Magn. Magn. Mater. 38, 34 (1983).

[2] E.I. Rashba, Fiz. Tverd. Tela 2, 1224 (1969).

[3] E.I. Rashba, V.I. Sheka, in: Landau Level Spectroscopy, Modern Problems in Condensed Matter Science, Vol. 27, Eds. G. Landwehr, E.I. Rashba, North-Holland, Amsterdam 1991, p. 131.

[4] M. Dobrowolska, H.D. Drew, J.K. Furdyna, T. Ichiguchi, A. Witowski, P.A. Wolff, Phys. Rev. Lett. 49, 845 (1982).

[5] M. Dobrowolska, Y. Chen, J.K. Furdyna, S. Rodriguez, Phys. Rev. Lett. 51, 134 (1983).

[6] M. Dobrowolska, A. Witowski, J.K. Furdyna, T. Ichiguchi, H.D. Drew, P.A. Wolff, Phys. Rev. B 29, 6652 (1984).

[7] M. Dobrowolska, Semicond. Sci. Technol. 5, 159 (1990).

[8] Z. Wilamowski, W. Jantsch, H. Malissa, U. Rössler, Phys. Rev. B 66, 195315 (2002).

[9] Z. Wilamowski, H. Malissa, W. Jantsch, private communication. 